\title{
7 Transgenic human gene reporter cell line for evaluating interactions between androgen receptor and xenobiotics
}

Bartonkova I, Novotna A, Dvorak Z

Department of Cell Biology and Genetics, Faculty of Science, Palacky University Olomouc, Slechtitelu 27, 78371 Olomouc

bartonkova.iveta@gmail.com,iveta.bartonkova@upol.cz

\section{Introduction:}

Androgen receptor (AR, NR3C4) is a 110-kDa ligand-activated transcriptional factor that belogs to the steroid hormone receptor superfamily. It has broad developmental and psychological effects and its mutations may have a great impact on pubertal development, genesis of prosthatic hyperplasia and even prostate cancer. Commonly known endogenous ligands of AR are testosterone and 5a-dihydrotestosterone (DHT). In the last years, several drugs and environmental pollutants were identified to alter AR activity leading to drug interactions or so called endocrine disruption. Therefore, development of in-vitro experimental tools for analyzing androgenic or antiandrogenic effects of various compounds is of great importance. In our work, we describe construction and characterization of a novel stably transfected human reporter cell line AIZ-AR for assessment of transcriptional activity of human androgen receptor. Cell line AIZ-AR is derived from human prostate carcinoma epithelial cell line 22Rv1 that was stably transfected with a reporter plasmid containing 3 copies of androgen response regions (ARRs) followed by a single copy of androgen response element (ARE) from the promoter region of human prostate specific antigen (PSA) gene. During the characterization process, our AIZ-AR cell line remained fully functional for over 60 days and more than 25 passages in the culture as well as after cryopreservation. Time-course analyses also revealed that AIZ-AR cells allow detection of AR ligands as soon as after 8 hours of the treatment. Upon dose-response analyses with 23 steroids in 96-well plate format, we observed induction of luciferase activity by androgens, but not by mineralocorticoids and estrogens. Some glucocorticoids and progesterone also activated AR, but with potencies 2-3 orders of magnitude lower as compared to androgens. Taken together, we have developed a rapid, sensitive selective, high-throughput and reproducible tool for detection of human AR ligands, with potential use in pharmacological and environmental applications.

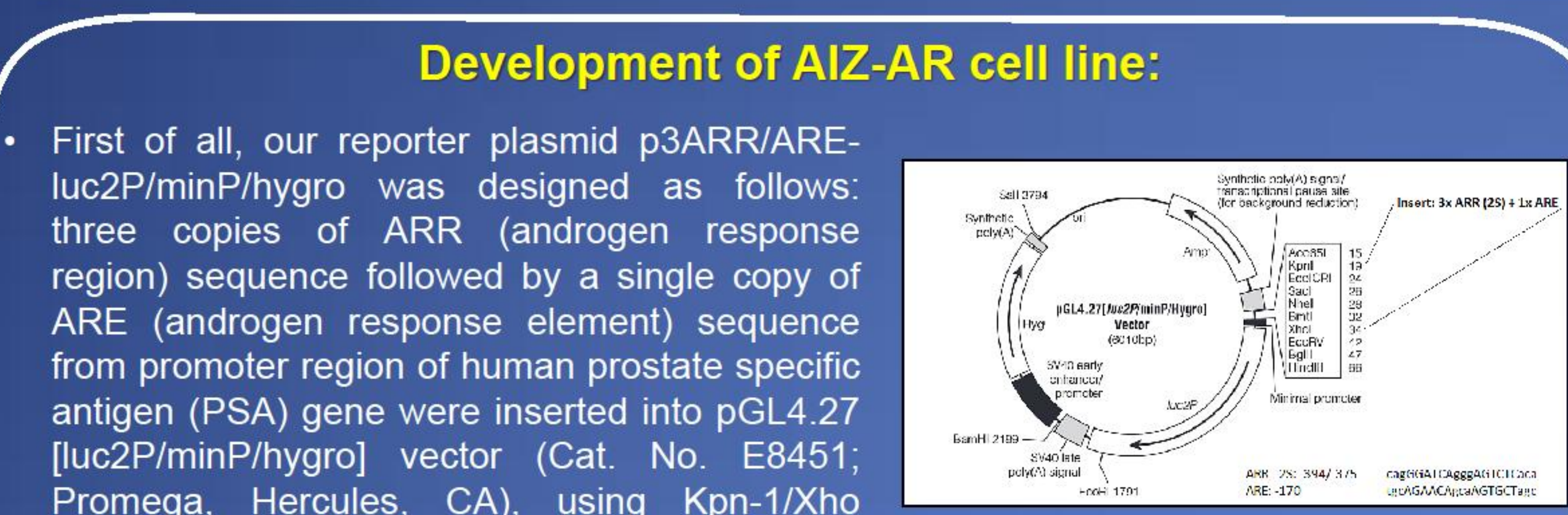

Promega, Hercules, CA), using Kpn-1/Xho restriction enzymes.

In the next step, human prostate carcinoma epithelial cell line 22Rv1 expressing fully functional endogenous androgen receptor was transfected with p3ARR/ARE reporter plasmid ( $4 \mu \mathrm{g}$ of DNA per $8 \times 10^{5}$ cells) using FugeneHD transfection reagent. Upon $48 \mathrm{~h}$ of stabilization, the medium was replaced with medium containing selection antibiotic. Stably transfected polyclonal population was selected within 3 weeks in the presence of hygromycin B $(0.5 \mathrm{mg} / \mathrm{ml})$.

Polyclonal population of stably transfected AIZ-AR cells was seeded for monoclonal population at density $10^{2}-10^{3}$ cells per Petri dish. After 2 weeks, clones were collected, subcultured and further characterized.

23 hygromycin-resistant clones obtained from the selection process were tested for responsivness to DHT. Cells were seeded in 96 -well plates at density $5 \times 10^{4}$ cells per well. Upon $16 \mathrm{~h}$ of stabilization, cells were treated with DMSO (vehicle, $0.1 \% \mathrm{v} / \mathrm{v}$ ) and DHT (100 $\mathrm{nM}$ ). After $24 \mathrm{~h}$, cells were lysed and luciferase activity was measured.
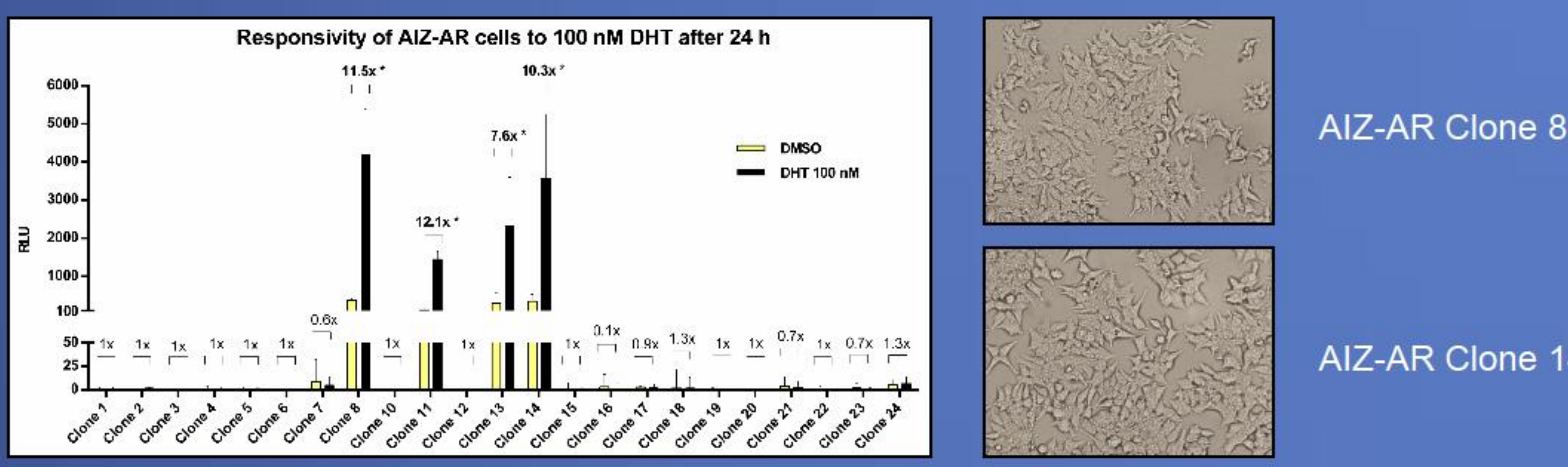

AIZ-AR Clone 14

Based on relative luciferase units, fold induction to DMSO and morphology similar to parental cell line 22Rv1, clones 8 and 14 were selected for further characterization and termed AIZ-AZ cells.

\section{Conclusion:}

- We have constructed stably transfected human cell line AIZ-AR that allows rapid, sensitive and high-throughput (96-well plate) screening of induction of AR transcriptional activity. - Human prostate carcinoma epithelial cell line 22Rv1 expressing fully functional endogenous androgen receptor was transfected with p3ARR/ARE reporter plasmid containing three copies of ARR (androgen response region) sequence followed by a single copy of ARE (androgen response element) sequence from promoter region of human prostate specific antigen (PSA) gene.

We obtained 4 clones inducible by $5 \alpha$-dihydrotestosterone (DHT) and selected 2 clones (clones 8 and 14) for further characterization and termed them AIZ-AR cells.

- During the characterization, AIZ-AR cells remained inducible for more than 25 passages (over 60 days) and even after cryopreservation.

- Time-course analyses of AIZ-AR cell line revealed the possibility of testing cytotoxic compounds, when 1000 RLU and 6-fold was obtained as soon as after 8 hours of incubation with DHT.

- Although some cross-reactivity with glucocorticoids might occur, our dose-response analyses show that AIZ-AR cell line can be used as highly selective method of identification of compounds with androgenic and antiandrogenic activity.

Taken together, we have developed entirely human, selective, rapid, high-throughput and reproducible stably transfected reporter system that can be used for various toxicological and environmental applications and drug discovery.
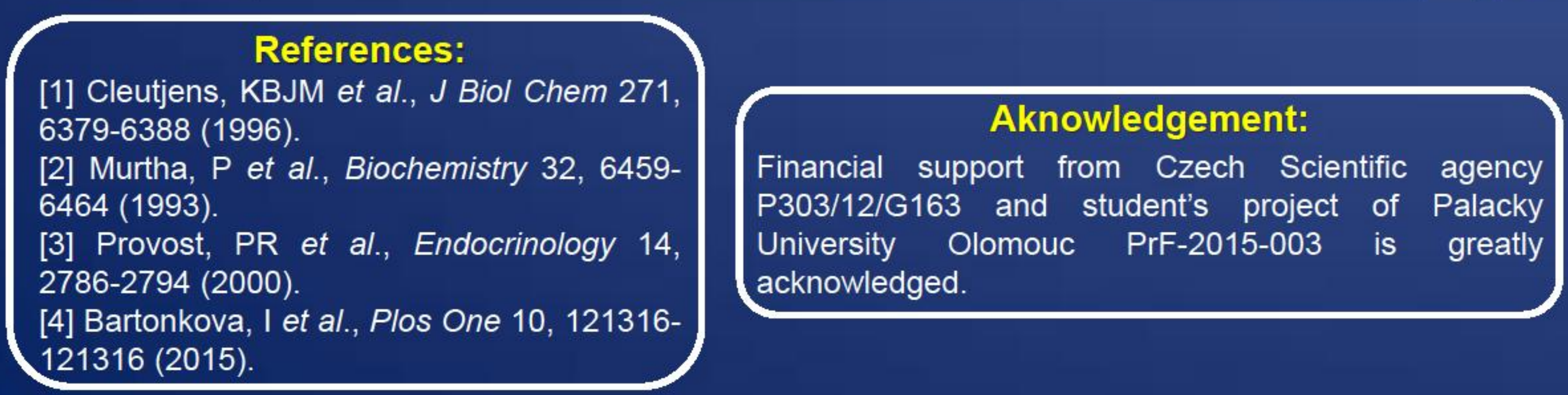

\section{Characterization of AIZ-AR cell line:}

First of all, we focused on maintenance of AIZ-AR cells functionality after cryopreservation. For this purpose, cells were frozen in fetal bovine serum and DMSO as cryoprotectant in ratio $9: 1$ and stored in $-80^{\circ} \mathrm{C}$ for 1 week. After thawing, both fresh and cryopreserved cells were seeded in 96-well plates and tested for inducibility after treatment with AR agonists (DHT, testosterone and danazol). No significant difference was observed between cryopreserved and fresh cells in terms of fold inductions and $\mathrm{EC}_{50}$ values. Only data for clone 8 are shown.

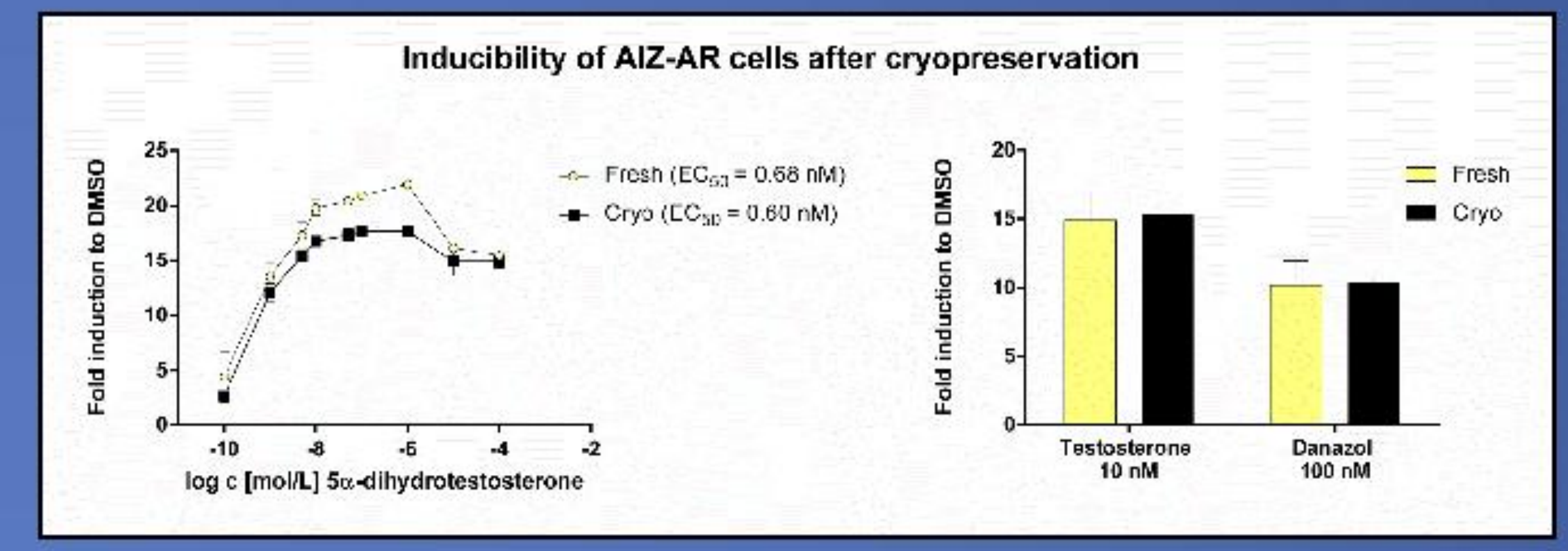

During the characterization, we tested long-term maintenance of inducibility of AIZ-AR cells after DHT treatment. Cells were seeded in 96 -well plates at density $5 \times 10^{4}$ cells per well in each passage. Upon $16 \mathrm{~h}$ of stabilization, cells were treated with DMSO $(0.1 \% \mathrm{v} / \mathrm{v})$ and DHT (100 nM). Our analyses revealed that AIZ-AR cell line remains fully functional for over 60 days in culture and for more than 25 passages.

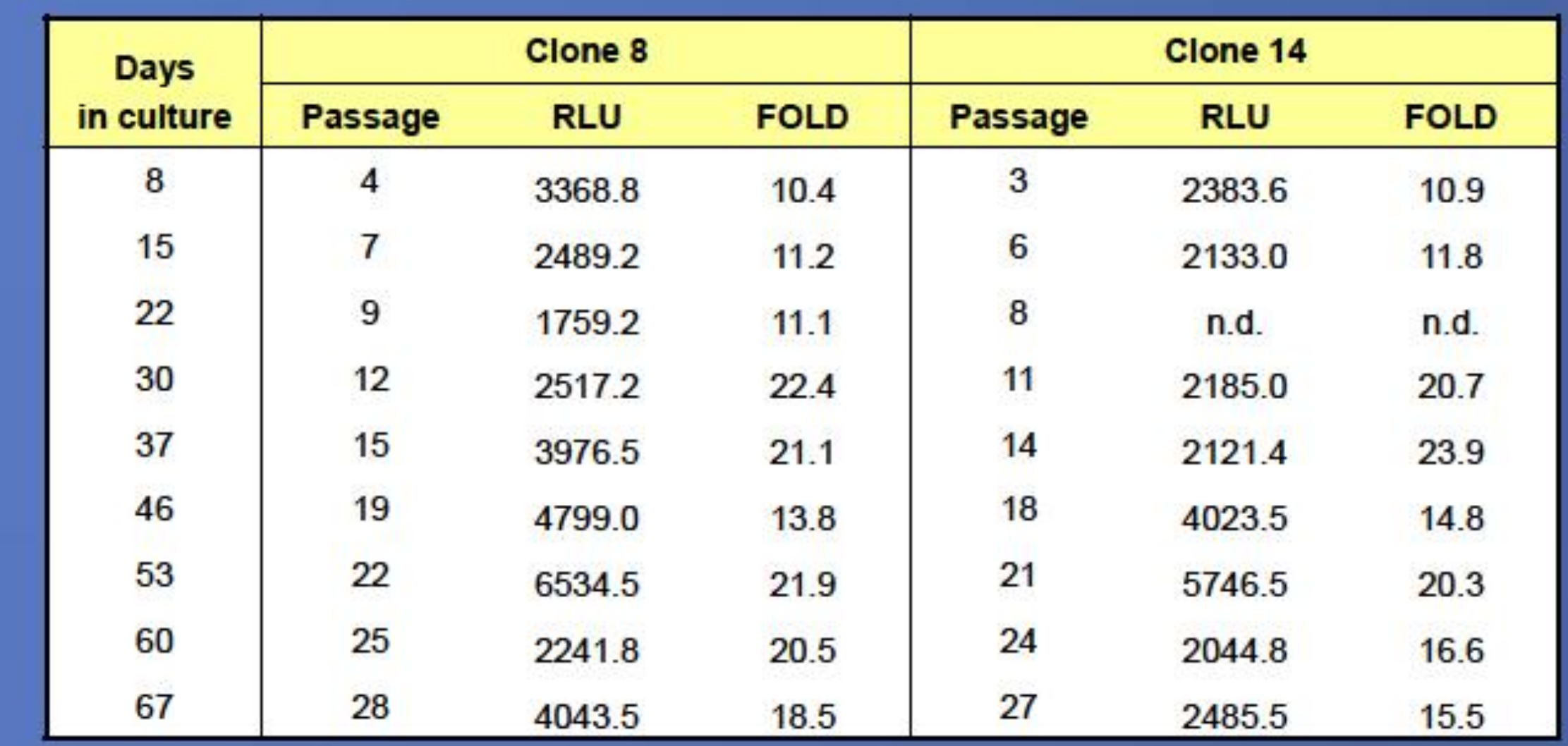

Subsequenty, we analyzed the time-course dependence of luciferase activity. Cells were seeded in 96-well plates and tested for inducibility after DHT treatment at intervals $2,4,6$ $8,10,12,14,16,18,20,22,24,48$ and 72 hours. Plateau in fold induction was attained approximately after $16 \mathrm{~h}$ of incubation. Luciferase activity around $1000 \mathrm{RLU}$ and induction about 6-fold was attained after 8 hours of incubation with DHT, implying the possibility to test cytotoxic compounds in $8 \mathrm{~h}$ time period. Only data for clone 8 are shown.

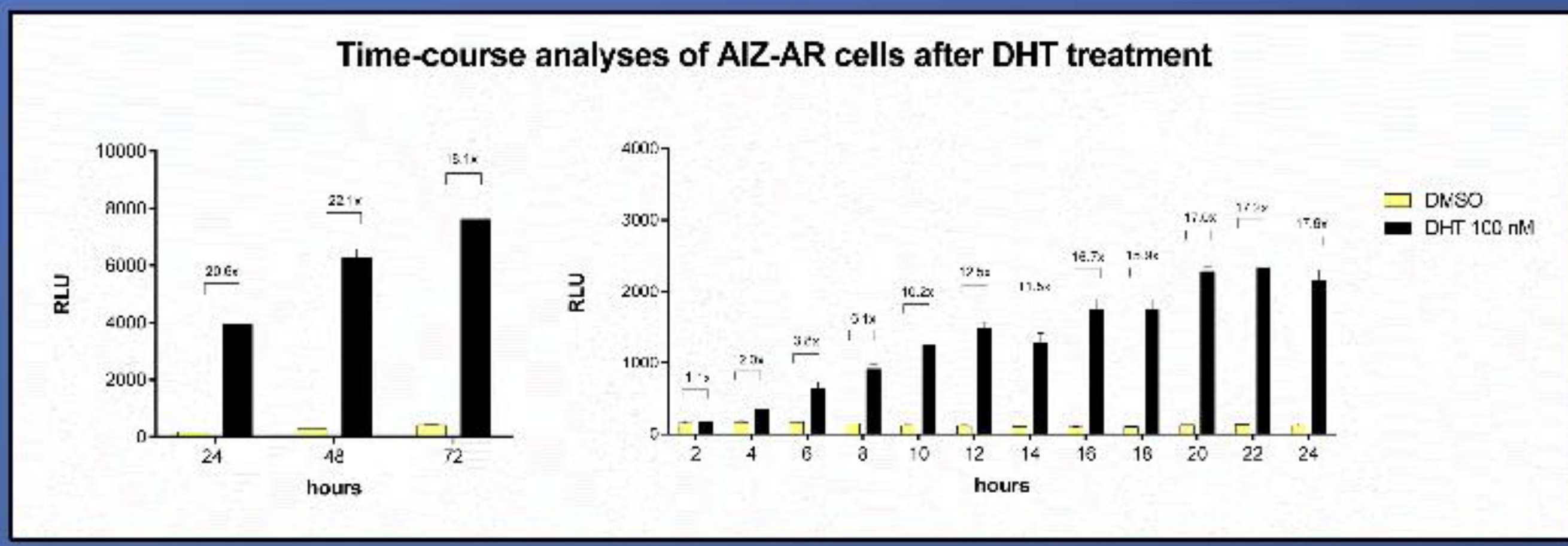

Finally, we focused on selectivity of AIZ-AR cells. We performed dose-response analyse of 23 steroid compounds. Cells were seeded in 96-well plates. After 16 hours of stabilization, cells were treated with tested compounds for 24 hours in the presence (antagonist mode) or absence (agonist mode) of model agonist DHT or testosterone. Ou data revealed that AIZ-AR cell line allows effective detection of compounds with androgenic activity. Some cross-reactivity with glucocorticoids occurs in concentrations of 2-3 orders of magnitude higher as compared to androgens. Only data for clone 8 are shown.

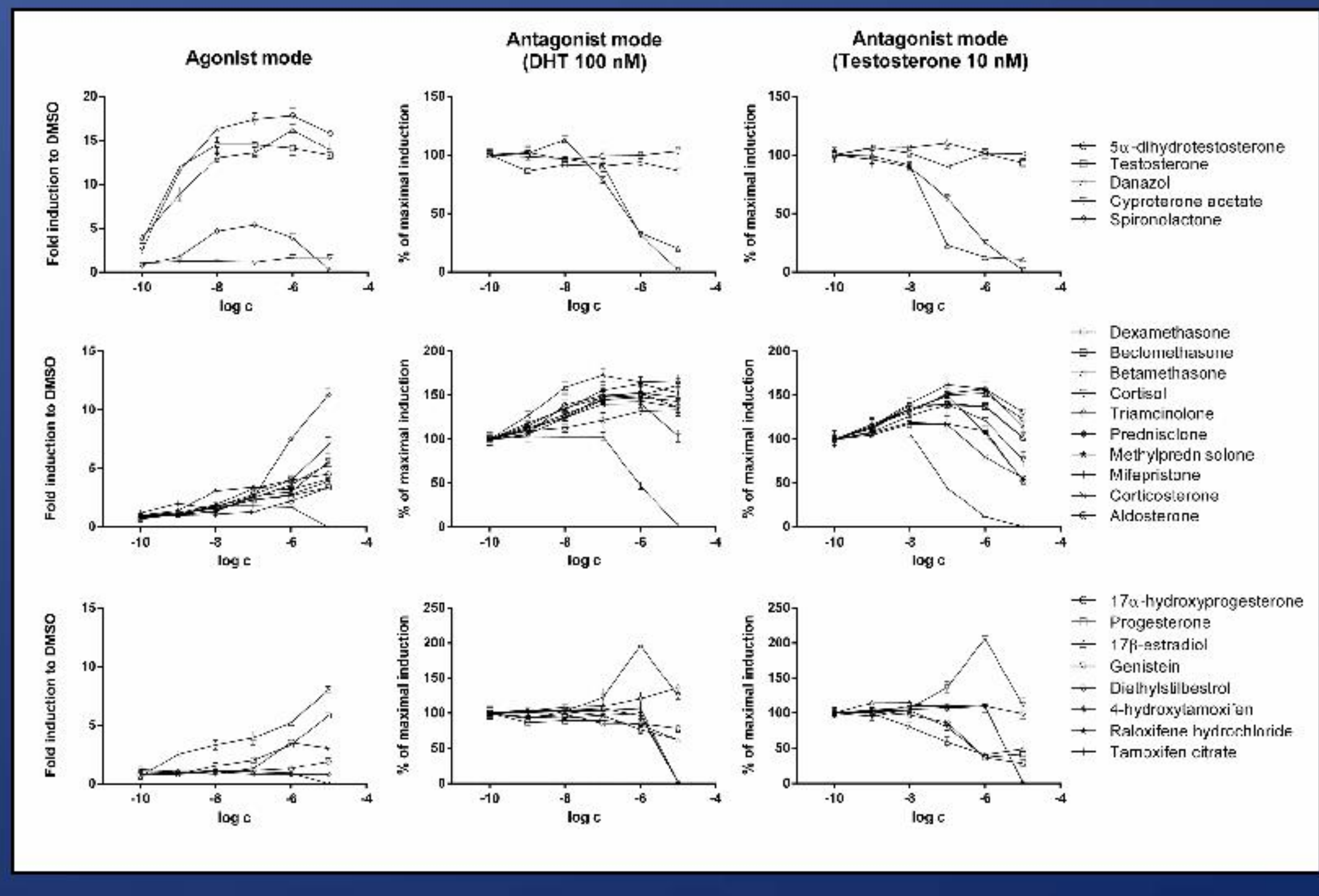

